\title{
Evaluation of Large Volume Srl2(Eu) Scintillator Detectors
}

B. W. Sturm, N. J. Cherepy, O. B. Drury, P. A. Thelin, S. E. Fisher, A. F. Magyar, S. A. Payne, A. Burger, L. A. Boatner, J. O. Ramey, K. S. Shah, R. Hawrami

November 19, 2010

IEEE Nuclear Science Symposium

Knoxville, TN, United States

October 30, 2010 through November 6, 2010 
This document was prepared as an account of work sponsored by an agency of the United States government. Neither the United States government nor Lawrence Livermore National Security, LLC, nor any of their employees makes any warranty, expressed or implied, or assumes any legal liability or responsibility for the accuracy, completeness, or usefulness of any information, apparatus, product, or process disclosed, or represents that its use would not infringe privately owned rights. Reference herein to any specific commercial product, process, or service by trade name, trademark, manufacturer, or otherwise does not necessarily constitute or imply its endorsement, recommendation, or favoring by the United States government or Lawrence Livermore National Security, LLC. The views and opinions of authors expressed herein do not necessarily state or reflect those of the United States government or Lawrence Livermore National Security, LLC, and shall not be used for advertising or product endorsement purposes. 


\title{
Evaluation of Large Volume $\mathrm{SrI}_{2}(\mathrm{Eu})$ Scintillator Detectors
}

\author{
Benjamin W. Sturm, Member, IEEE, Nerine J. Cherepy, Member, IEEE, Owen B. Drury, Peter A. Thelin, Scott E. \\ Fisher, Albert F. Magyar, Stephen A. Payne, Member, IEEE, Arnold Burger, Member, IEEE, Lynn A. Boatner, \\ Member, IEEE, Joanne O. Ramey, Kanai S. Shah, Member, IEEE, Rastgo Hawrami, Member, IEEE
}

\begin{abstract}
There is an ever increasing demand for gamma-ray detectors which can achieve good energy resolution, high detection efficiency, and room-temperature operation. We are working to address each of these requirements through the development of large volume $\mathrm{SrI}_{2}(\mathrm{Eu})$ scintillator detectors. In this work, we have evaluated a variety of $\mathrm{SrI}_{2}$ crystals with volumes $>10 \mathrm{~cm}^{3}$. The goal of this research was to examine the causes of energy resolution degradation for larger detectors and to determine what can be done to mitigate these effects. Testing both packaged and unpackaged detectors, we have consistently achieved better resolution with the packaged detectors. Using a collimated gamma-ray source, it was determined that better energy resolution for the packaged detectors is correlated with better light collection uniformity. A number of packaged detectors were fabricated and tested and the best spectroscopic performance was achieved for a 3\% Eu doped crystal with an energy resolution of $2.93 \%$ FWHM at $662 \mathrm{keV}$. Simulations of $\mathrm{SrI}_{2}(\mathrm{Eu})$ crystals were also performed to better understand the light transport physics in scintillators and are reported. This study has important implications for the development of $\mathrm{SrI}_{2}(\mathrm{Eu})$ detectors for national security purposes.
\end{abstract}

\section{INTRODUCTION}

$T$ HE recent discovery that $\mathrm{SrI}_{2}(\mathrm{Eu})$ can provide energy resolution comparable to that of $\mathrm{LaBr}_{3}(\mathrm{Ce})$ offers a new option for gamma-ray detection and spectroscopy [1, 2]. Some properties of the $\mathrm{SrI}_{2}(\mathrm{Eu})$ scintillator that make it desirable for gamma-ray detection include: a high light yield of $\sim 90,000$ photons/MeV, excellent light yield proportionality, an emission band of $410-450 \mathrm{~nm}$ that is well-matched to bialkali PMTs, and no intrinsic radioactivity [2-5]. Small $\mathrm{SrI}_{2}(\mathrm{Eu})$ crystals of $\sim 1 \mathrm{~cm}^{3}$ in volume have demonstrated $<2.7 \%$ resolution at $662 \mathrm{keV} \mathrm{[6].} \mathrm{Until} \mathrm{recently,} \mathrm{large} \mathrm{crystals}$ with volumes $>10 \mathrm{~cm}^{3}$ generally provided poorer resolution. This paper focuses on how the optical light trapping effect,

Manuscript received November 19, 2010. This work was supported by the Department of Homeland Security Domestic Nuclear Detection Office and was performed under the auspices of the U.S. Department of Energy by Lawrence Livermore National Laboratory under Contract DE-AC5207NA27344.

B. W. Sturm, N. J. Cherepy, O. B. Drury, P. A. Thelin, S. E. Fisher, A. F. Magyar, and S. A. Payne are with Lawrence Livermore National Laboratory, Livermore, CA 94550 USA. (corresponding author e-mail: sturm1 @1lnl.gov).

A. Burger is with Fisk University, Nashville, TN 37201 USA.

L. A. Boatner and J. O. Ramey are with Oak Ridge National Laboratory, Oak Ridge, TN 37830 USA.

K. S. Shah and R. Hawrami are with Radiation Monitoring Devices, Watertown, MA 02472 USA. due to re-absorption and re-emission by Eu in the crystal, can be mitigated by proper geometry and optical design.

\section{Evaluating Resolution DEgRADATION IN LARGE VOLUME CRYSTALS}

The fact that $\mathrm{SrI}_{2}(\mathrm{Eu})$ exhibited excellent spectroscopic performance with small crystals $\left(\sim 1 \mathrm{~cm}^{3}\right)$ implies that intrinsically this material is very good as a gamma-ray detection material. The following sections discuss experiments that were conducted to diagnose the performance degradation seen in large volume $\left(>10 \mathrm{~cm}^{3}\right)$ crystals.

\section{A. Collimation Study of an Unpackaged Crystal}

A technique was developed to diagnose the problem of high energy tailing observed in $\mathrm{SrI}_{2}(\mathrm{Eu})$ involving a collimated gamma-ray source. This was accomplished using the method depicted in Fig. 1a. Here, two lead bricks were positioned to form a slit for collimating ${ }^{137} \mathrm{Cs}$ gamma-rays. The collimated beam was then scanned along different slices of the crystal horizontal to the PMT window. This allowed us to probe the scintillator response as a function of interaction position. The results of such a scan for one particular large volume crystal are shown in Fig. 1b. This figure represents the spectrum acquired at each of the collimation regions. Here we see a clear trend in terms of increasing photopeak position, and thus higher light collection, as the scan distance (z) decreases. This means that a greater fraction of the light is collected at the bottom of the crystal (pos4) than at the top (pos1). This trend is believed to be caused by a combination of light trapping, as well as light losses at the surface of the crystal. Another trend that we typically observe is poorer resolution as the gammaray interaction occurs closer to the PMT window. The cause of this energy resolution degradation will be discussed further in Section IV.B.

\section{B. Packaging Study}

The hygroscopic nature of $\operatorname{SrI}_{2}(\mathrm{Eu})$ means that it is necessary to package this material in a hermetically sealable canister. To better understand the effects of packaging $\mathrm{SrI}_{2}$, a 

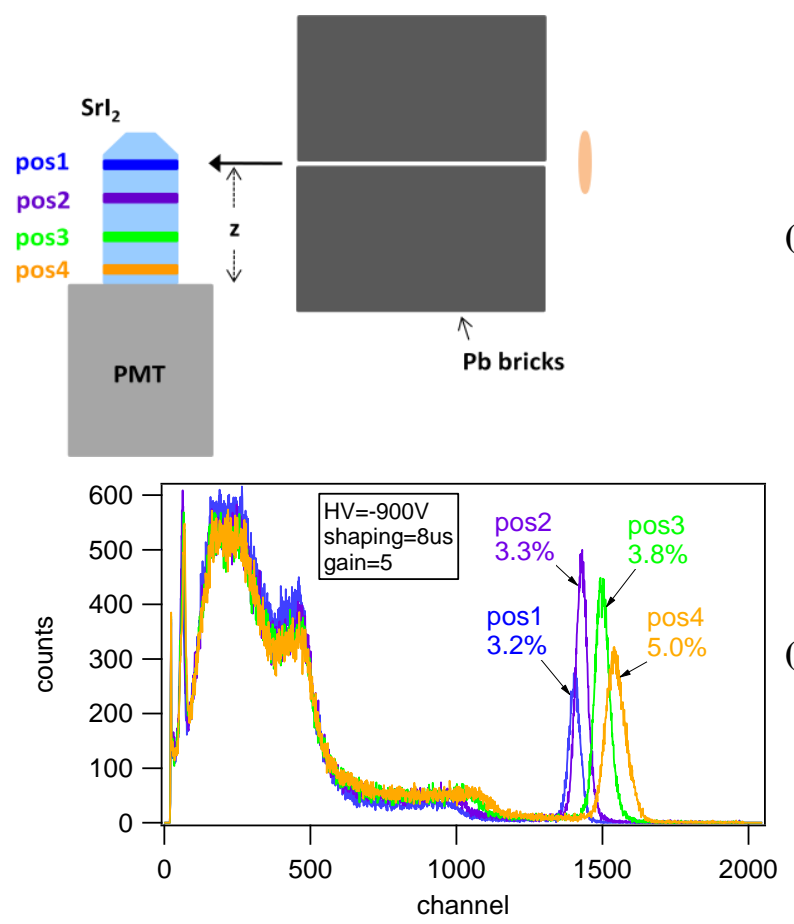

Fig. 1. Collimation experiment to diagnose high energy tailing of large crystals. Fig. 1(a) shows a schematic of the experimental setup and 1(b) are the resulting spectra of a crystal with a volume of $19.7 \mathrm{~cm}^{3}$. We see a clear trend in which the photopeak centroid position increases with decreasing scan distance from the PMT window, while the resolution (percent value indicated below position) degrades. The peak shift between position 1 and position 4 is $\sim 10 \%$.

study was done by incorporating each component of the packaging process separately and observing the change in detector performance for each step. In step 1, a bare tapered cylinder crystal was wrapped with 4 layers of Teflon tape and then placed on the PMT with a thin layer of mineral oil acting as a light coupling agent. The entire crystal and PMT assembly was then tented with 6 additional layers of Teflon tape. In step 2, a $1 \mathrm{~mm}$ thick BK7 glass window between the crystal and the PMT was incorporated. In this case, silicone optical grease (BC-630) was used as the coupling compound. Finally, in step 3, two additional layers of Teflon were wrapped around the crystal and the packaging canister was then incorporated. This final step emulates the fully packaged crystal. Pulse height spectra were recorded for each of these steps and the results are shown in Fig. 2.

The spectra in Fig. 2 show a reduction in the photopeak centroid between step 1 and step 2. This indicates reduced light collection efficiency by incorporating the $1 \mathrm{~mm}$ thick glass window. Although less light was collected, the energy resolution improved slightly from $4.36 \%$ to $4.09 \%$ FWHM at $662 \mathrm{keV}$. Then, step 3 gives rise to a small increase in light collection efficiency (after step 2) and a dramatic improvement in energy resolution, with a final value of $3.18 \%$. This result indicates that the packaging method implemented actually helps to improve energy resolution.

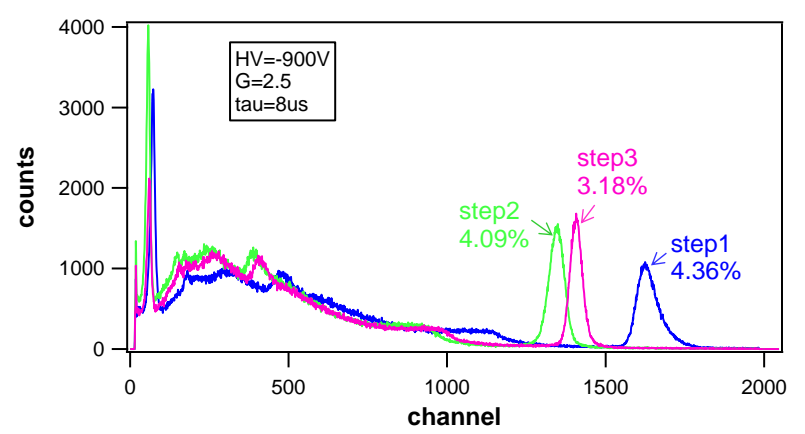

Fig. 2. Packaging study done on a $5 \%$ Eu doped tapered cylinder crystal with a volume of $10.3 \mathrm{~cm}^{3}$. Step 1 is a ${ }^{137} \mathrm{Cs}$ spectrum taken with the crystal wrapped with 4 layers of Teflon tape and placed directly on the PMT, step 2 is with the crystal on a $1 \mathrm{~mm}$ thick BK7 glass window, and step 3 is with 2 more layers of Teflon and incorporating the $\mathrm{Al}$ canister. There is a clear improvement in energy resolution (percent value indicated below position) between step 1 and step 3, indicating that packaging actually helps to improve performance.

\section{Collimation Study of Crystal Before and After Packaging}

To demonstrate why we see improved energy resolution after packaging, collimation measurements were acquired of the same crystal before and after packaging. The results of which are displayed in Fig. 3. Fig. 3(a) gives the collimated spectra for the unpackaged crystal and Fig. 3(b) is for the packaged crystal. For the unpackaged crystal there is a photopeak shift of $3.2 \%$ between the top and the bottom of the crystal, whereas for the packaged crystal the photopeak shift reduces to $1.3 \%$. The reduced magnitude of photopeak shift for the packaged crystal indicates that there is better light collection uniformity. This leads to better energy resolution, since the number of photons arriving at the photocathode will be less dependent on the position of the gamma-ray interaction. A discussion of these results is expanded futher in [6].

\section{RESUlTS FROM NEWLY PACKAGED CRYSTALS}

\section{A. $\mathrm{SrI}_{2}$ Crystals Doped with $5 \% \mathrm{Eu}$}

Our recent set of crystals were packaged using the version 3 packaging design as described in [6]. The spectrum of one such detector with a volume of $10.3 \mathrm{~cm}^{3}$ gave rise to $3.22 \%$ FWHM energy resolution at $662 \mathrm{keV}$ as shown in Fig. 4. Two other 5\% Eu-doped packaged crystals resulted in $3.30 \%$ and $2.99 \%$ energy resolution with volumes of $11.2 \mathrm{~cm}^{3}$ and 10.3 $\mathrm{cm}^{3}$, respectively. For all cases the energy resolution for the packaged crystal was significantly better than for the unpackaged crystal. 


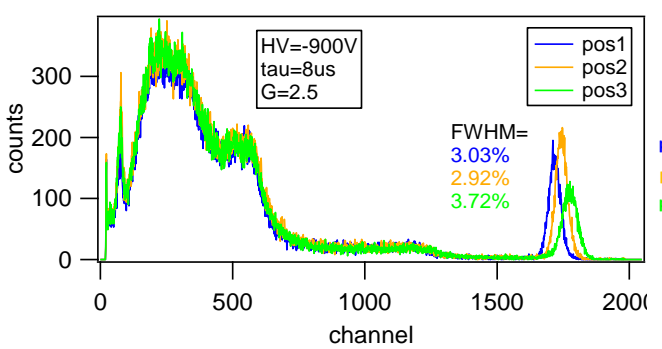

(a)
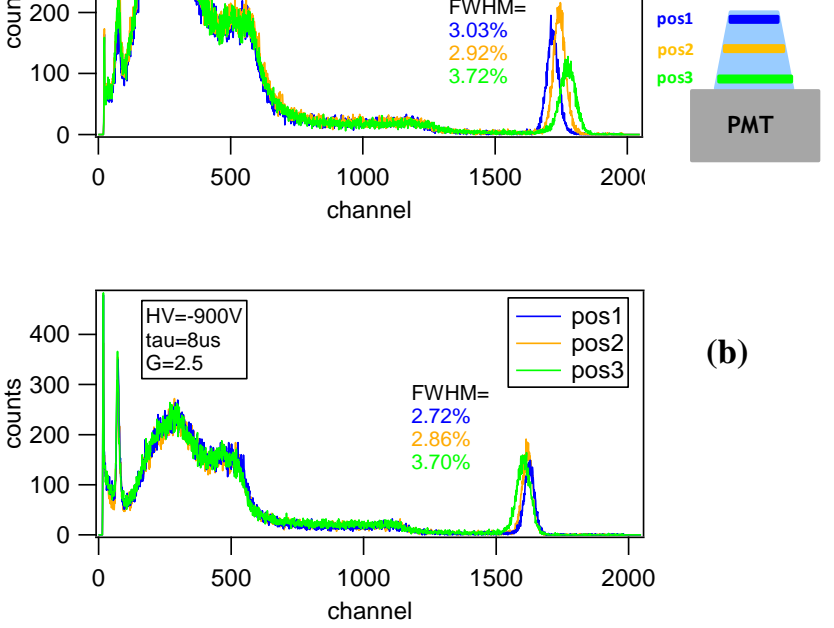

(b)

Fig. 3. Collimation results of the same crystal for the unpackaged (a) and packaged (b) case. For the unpackaged crystal, the total photopeak shift was $3.2 \%$, resulting in an overall energy resolution of 5.01\% FWHM at $662 \mathrm{keV}$. For the packaged crystal, the total photopeak shift was $1.3 \%$, which resulted in a significant improvement in energy resolution with a value of $3.22 \%$.

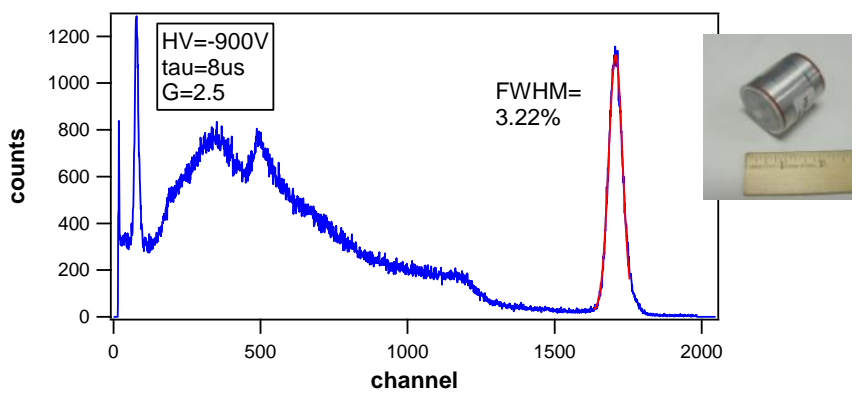

Fig. 4. ${ }^{137} \mathrm{Cs}$ spectrum of a $5 \% \mathrm{Eu}$ doped crystal using the version 3 packaging design. This crystal has a volume of $10.3 \mathrm{~cm}^{3}$.

\section{B. $\mathrm{SrI}_{2}$ Crystal Doped with $3 \% \mathrm{Eu}$}

To test the effect of dopant concentration on energy resolution, a 3\% Eu-doped crystal was grown by RMD. A 1x1 inch $^{2}$ cylinder was cut from the boule and formed into a tapered cylinder geometry and subsequently polished. The final crystal volume was $13.2 \mathrm{~cm}^{3}$. A ${ }^{137} \mathrm{Cs}$ spectrum was recorded of the unpackaged crystal and a resolution of $3.47 \%$ FWHM was measured. This result was the best we have recorded for an unpackaged large volume crystal. To understand this result better, collimation measurements were acquired and the resulting spectra are shown in Fig. 5. Here we see that there is relatively little peak shift $(1.8 \%)$ between pos 1 and pos4. The reduced peak shift in comparison to the 5\% Eu-doped case shown in Fig. 3(a), may be related to less light trapping in the crystal with the smaller Eu concentration [7]. Another effect we see in Fig. 5 is that the energy resolution at any individual depth is very good, ranging from $2.82 \%$ to $3.13 \%$. The variation in energy resolution at any particular depth is less severe than in the 5\% Eu doped

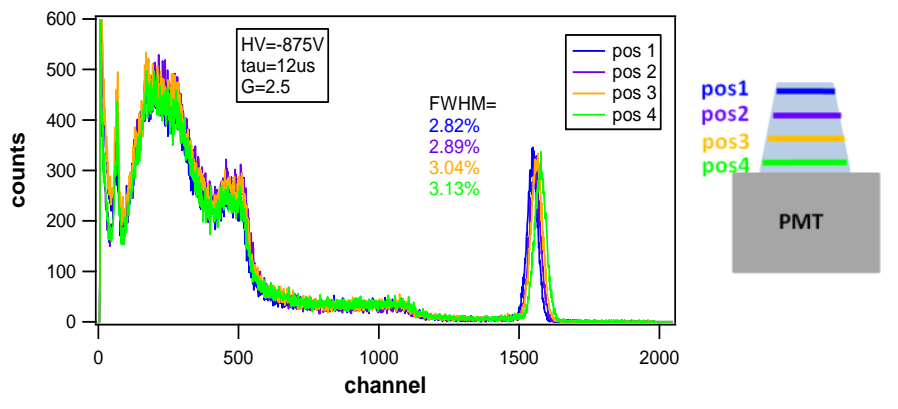

Fig. 5. Collimation results of a $3 \%$ Eu doped unpackaged detector with a volume of $13.2 \mathrm{~cm}^{3}$. The total peak shift was $1.8 \%$, indicating that the light collection uniformity for the $3 \%$ Eu doped crystal may be superior to that of the $5 \%$ Eu doped crystal.

sample, which again may be related to the effect of less light trapping in the crystal.

This crystal was then packaged using the version 3 packaging design and a ${ }^{137} \mathrm{Cs}$ pulse height spectrum was recorded as shown in Fig. 6. Here, we achieved an energy resolution of 2.93\% FWHM at $662 \mathrm{keV}$, which is the best we have recorded for a large volume detector. From these results as well as the unpackaged crystal results, it seems that $3 \% \mathrm{Eu}$ doping may be the preferred concentration for achieving the best detector performance.

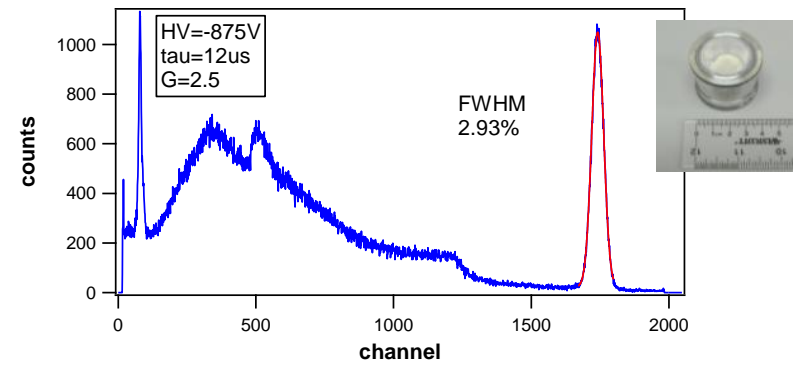

Fig. 6. ${ }^{137} \mathrm{Cs}$ spectrum recorded on the packaged $3 \%$ Eu doped crystal. This is the best resolution ever obtained on a large volume $\operatorname{SrI}_{2}(\mathrm{Eu})$ detector.

\section{SIMULATION STUDY TO UNDERSTAND LIGHT TRANSPORT PHYSICS IN SRI 2 (EU)}

To better understand the light transport physics in $\mathrm{SrI}_{2}(\mathrm{Eu})$ radiation detectors, simulations were performed using the Monte-Carlo code DETECT2000 [8,9]. This code is very useful for modeling the optical behavior in scintillator detectors. We assumed some basic input parameters in our model, including a bulk absorptive length of $1000 \mathrm{~mm}$ and a refractive index of 2.05. We implemented the UNIFIED surface model, which is a feature of DETECT2000, allowing the user to model various scintillator surface roughnesses, the reflectivity of an external diffuse reflector, as well as the index of refraction of the material separating the reflector from the scintillator. A specular lobe finish was specified with a sigmaalpha value of $1.3^{\circ}$. An external diffuse reflector was also modeled with a reflection coefficient of 0.99 . These values have been verified to be good approximations of a mechanically polished surface wrapped with Teflon tape [10]. 
To accurately model the response of these detectors, it is not only important to model the crystal and reflector parameters, but also the emission spectrum of the scintillator as well as the absorption spectrum of the photodetector. Hence, in these simulations, the $\mathrm{SrI}_{2}(\mathrm{Eu})$ emission spectrum as well as the photocathode quantum efficiency versus photon wavelength spectrum were incorporated. Finally, a tapered cylinder crystal geometry was specified $1 \mathrm{x} 1$ inch $^{2}$ with a top diameter of $3 / 4$ inch.

\section{A. Simulation of the Collimation Experiment Peak Shift}

To better understand the photopeak shift observed in the collimation experiment, simulations were done to see if this effect could be reproduced. In DETECT2000, one can effectively simulate the collimation experiment by generating scintillation photons in different regions of the crystal. In these simulations, $10^{6}$ photons were generated in 4 different regions, corresponding to the different scan slices. Then, the fraction of the photons that get detected by the photocathode were recorded. Simulations were done for three different optical scatter lengths, $15 \mathrm{~mm}, 25 \mathrm{~mm}$, and $40 \mathrm{~mm}$. The optical scatter length is the mean distance the photons travel before being scattered and redirected isotropically. This is a reasonably good first approximation of the effect of light trapping, where the photon gets absorbed and reemitted by the $\mathrm{Eu}^{2+}$ [7]. The results of this simulation are shown in Fig. 7. We found that the case of $15 \mathrm{~mm}$ optical scatter length reproduced our data very well. For this case the total simulated peak shift between pos 1 and pos 4 was $3.75 \%$, which agrees well with the experimental data shown in Fig. 3a. Here, the peak shift for a 5\% Eu doped sample was 3.2\%. In Fig. 7 we also find that the trend is the same as the experimental data in that the most light is collected at position 4 (bottom) and the least light is collected at position 1 (top). We therefore used a $15 \mathrm{~mm}$ optical scatter length input parameter for the simulations in the next section.

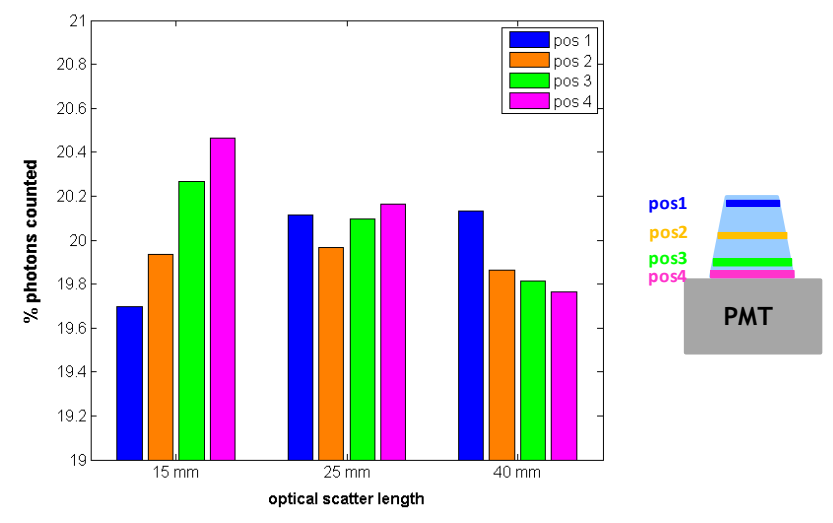

Fig. 7. Simulation results using DETECT2000 of the \% photons counted by the photocathode for each of the photon generation regions. Simulations were done for 3 different optical scatter lengths and it was found that the $15 \mathrm{~mm}$ setting reproduced the experimental data very well. For this case, the total simulated peak shift was $3.75 \%$. In addition, the trend of increased light collection as the scan distance decreases (closer proximity to the PMT) is the same.
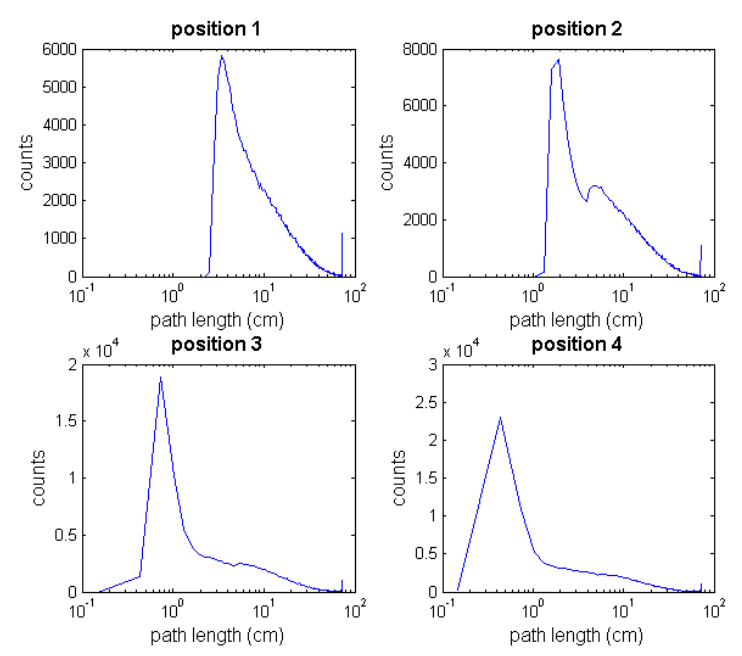

Fig. 8. Simulation results of the photon path length histograms for different regions of the crystal (see Fig. 7). We find that there is greater dispersion in the photon path lengths the closer the scintillation photons are generated to the PMT window. Greater dispersion will translate to poorer energy resolution.

\section{B. Simulations to Model Photon Path Length Distributions}

In section II.A, it was found that the energy resolution of an unpackaged crystal generally degrades as the scan distance, $\mathrm{z}$, decreases (Fig. 1). To model this effect, photons were generated in the different scan regions (as described in the previous section), and the distribution of the photon path lengths for each of these regions were plotted. The results are shown in Fig. 8. For position 1 (top left plot), which corresponds to the top of the crystal, the photons generated in this region will all be directed toward the PMT and therefore the path length distribution is a simple exponential decay function governed by the absorption mean free path setting. For position 2 (top right plot), however, one can imagine that about half of the photons will be directed toward the PMT and half will be directed away. This will result in a bimodal distribution of the photon path lengths as seen by the double peak feature. This effect becomes accentuated the closer the scintillation photons interact to the PMT window and thus we see greater dispersion in the path length distribution for position 3 (bottom left) and position 4 (bottom right). Greater dispersion will mean greater variation in the photons detected by the photocathode, which will give rise to poorer energy resolution. Although these effects are a source of energy resolution degradation in $\mathrm{SrI}_{2}(\mathrm{Eu})$ detectors, it is important to note that such observations can only be made due to the intrinsically superior energy resolution observed in this material.

\section{CONCLUSIONS}

This paper describes the performance and characteristics of large volume $\left(>10 \mathrm{~cm}^{3}\right) \mathrm{SrI}_{2}(\mathrm{Eu})$ detectors. Measurements with a collimated ${ }^{137} \mathrm{Cs}$ source helped to diagnose the effect of nonuniform light collection in $\mathrm{SrI}_{2}(\mathrm{Eu})$ crystals. It was found that the photopeak shift between collimation scans at the top 
and bottom of the crystal could be reduced from $3.2 \%$ for the unpackaged crystal to $1.3 \%$ for the packaged crystal. Reduced peak shifting means better light collection uniformity, ultimately leading to better energy resolution. Results for four different packaged crystals were reported and although very good energy resolution was achieved for the 5\% Eu doped samples $(3.0 \%-3.3 \%$ FWHM at $662 \mathrm{keV})$, the best energy resolution of $2.93 \%$ was reported for the $3 \%$ Eu doped crystal. This may be due to less light trapping in the smaller Eu concentration sample. Lastly, simulations using DETECT2000 were discussed and they provided insight into the mechanism of the light transport physics in $\operatorname{SrI}_{2}(\mathrm{Eu})$ scintillators. We found good agreement between simulation and experiment both for the photopeak shift as well as the energy resolution trends as a function of interaction position.

\section{ACKNOWLEDGMENT}

The authors would like to thank Martin Janecek of Lawrence Berkeley National Laboratory for his help and advice with regard to the DETECT2000 simulations.

\section{REFERENCES}

[1] R. Hofstadter, "Europium Activated Strontium Iodide Scintillators," US Patent No. 3, 373, 279, Mar. 12,1968.

[2] N. J. Cherepy, G. Hull, A. D. Drobshoff, S. A. Payne, E. v. Loef, C. M. Wilson, K. S. Shah, U. N. Roy, A. Burger, L. A. Boatner, W.-S. Choong, and W. W. Moses, "Strontium and barium iodide high light yield scintillators," Applied Physics Letters, vol. 92, pp. 083508, 2008.

[3] N. J. Cherepy, B. W. Sturm, O. B. Drury, T. A. Hurst, S. A. Sheets, L. E. Ahle, C. K. Saw, M. A. Pearson, S. A. Payne, A. Burger, L. A. Boatner, J. O. Ramey, E. V. van Loef, J. Glodo, R. Hawrami, W. M. Higgins, K. S. Shah, and W. W. Moses, "SrI 2 scintillator for gamma ray spectroscopy," Proceedings of the SPIE - The International Society for Optical Engineering, vol. 7449, pp. 74490F (6 )-74490F (6 ), 2009.

[4] N. J. Cherepy, S. A. Payne, S. J. Asztalos, G. Hull, J. D. Kuntz, T. Niedermayr, S. Pimputkar, J. J. Roberts, R. D. Sanner, T. M. Tillotson, E. van Loef, C. M. Wilson, K. S. Shah, U. N. Roy, R. Hawrami, A. Burger, L. A. Boatner, C. Woon-Seng, and W. W. Moses, "Scintillators with potential to supersede lanthanum bromide," IEEE Transactions on Nuclear Science, vol. 56, pp. 873-880, 2009.

[5] R. Hawrami, M. Groza, Y. Cui, A. Burger, M. D. Aggarwal, N. Cherepy, and S. A. Payne, "SrI 2 : a novel scintillator crystal for nuclear isotope identifiers," presented at Hard X-Ray, Gamma-Ray, and Neutron Detector Physics X, 2008.

[6] B. W. Sturm, N. J. Cherepy, O. B. Drury, P. A. Thelin, S. E. Fisher, S A. Payne, A. Burger, L. A. Boatner, J. O. Ramey, K. S. Shah, and R. Hawrami, "Effects of packaging SrI2(Eu) scintillator crystals," Nuclear Instruments and Methods in Physics Research Section A: Accelerators, Spectrometers, Detectors and Associated Equipment, vol. In Press, Corrected Proof, 2010.

[7] J. Glodo, E. V. van Loef, N. J. Cherepy, S. A. Payne, and K. S. Shah, "Concentration Effects in Eu Doped $\mathrm{SrI}_{2}$, " Nuclear Science, IEEE Transactions on, vol. 57, pp. 1228-1232, 2010.

[8] G. F. Knoll, T. F. Knoll, and T. M. Henderson, "Light collection in scintillation detector composites for neutron detection," Nuclear Science, IEEE Transactions on, vol. 35, pp. 872-875, 1988.

[9] F. Cayouette, D. Laurendeau, and C. Moisan, "DETECT2000: an improved Monte-Carlo simulator for the computer aided design of photon sensing devices," SPIE Proceedings, vol. 4833, pp. 69-76, 2003.

[10] M. Janecek and W. W. Moses, "Simulating Scintillator Light Collection Using Measured Optical Reflectance," Nuclear Science, IEEE Transactions on, vol. 57, pp. 964-970, 2010 